\title{
Cytomorphological Analysis of Keratinocytes in Oral Smears from Tobacco Users and Oral Squamous Cell Carcinoma Lesions - A Histochemical Approach
}

\author{
Suneet Khandelwal ${ }^{1}$, Monica Charlotte Solomon ${ }^{2 *}$ \\ ${ }^{1}$ Jaipur Dental College, Jaipur Highway, Jaipur, India \\ ${ }^{2}$ Department of Oral Pathology, Manipal College of Dental Sciences, Manipal University, Manipal, India
}

\begin{abstract}
Aim To analyse the cytomorphological features of keratinocytes in smears obtained from the oral mucosa of tobacco users and from oral squamous cell carcinoma lesions.

Methodology Oral smears were obtained from clinically, normal appearing mucosa of oral squamous cell carcinoma patients $(n=20)$ and from the mucosa of smokers $(n=20)$, and apparently healthy individuals $(n=20)$ were used as controls. The smears were histochemically stained and cytomorphological assessment of the keratinocytes was carried out. One-way ANOVA (Analysis of Variance) was used for comparing the parameters among multiple groups and Tukey-HSD test was used to compare the mean values
\end{abstract}

between groups.

Results The mean nuclear area of keratinocytes from the mucosa of tobacco users was $46 \pm 2.57$ and that of the oral squamous cell carcinoma lesion was $81.54 \pm 4.31$. While there was a significant $(P=0.001)$ reduction in the cellular area of keratinocytes from oral squamous cell carcinoma lesion when compared with those from oral smears of tobacco users.

Conclusion Cytomorphometric analysis of keratinocytes can serve as a useful adjunct in the early diagnosis of oral squamous cell carcinomas.

Keywords exfoliative cytology, cytomorphometry, Feulgen stain, tobacco users, oral cancer

Received Jan. 12, 2010; Revision accepted Feb. 20, 2010

\section{Introduction}

Oral cancer is currently the sixth most common malignancy in the world (Parkin et al., 2000). In India it is the most common malignancy among men and one of the five most common malignancies among women (Boyle et al., 1989). The etiology of oral squamous cell carcinoma (OSCC) is multifactorial, but tobacco usage in various forms continues to be an important risk factor (Ramesh et al., 1999): Despite advances in surgery, radiotherapy and chemotherapy, the 5-year survival rate of OSCC patients has remained approximately $50 \%$ (SEER, 1998). This poor survival rate among oral cancer patients can be attributed to several factors; one prominent factor being the lack of early detection and treatment. A key approach to this problem would be to detect potentially malignant lesions at their earliest or incipient stage.

As per the normal physiology, the oral epithelium renews itself rapidly (probably every 2 weeks). The rationale of oral exfoliative cytology is based on this physiological process, examining cells that are desquamated or abraded from the surface of the oral mucosa. Miller et al. were the first to study the cytology of the normal oral epithelium (Miller et al., 1951). The superficial epithelial cells do contain nuclei, and thus, alterations in these cells can serve as reliable indicators of 
dysplastic or neoplastic changes. Montogomery and his associates applied the principle of exfoliative cytology for the diagnosis of oral cancer (Montogomery et al., 1951). Later, Cowpe et al. pointed out that the application of quantitative techniques to cytology could markedly improve its diagnostic sensitivity (Cowpe et al., 1981).

The Papanicolau stain is regarded as the universal stain for cytological preparations. This special stain imparts a different colour to the cytoplasm of the epithelial cells based on their degree of cellular differentiation (Sivapathasundaram et al., 2004). Other histochemical stains have also been used for oral smears, and one of them is the Feulgen stain (Bancroft et al., 1996; Sugerman et al., 1996). The Feulgen stain is one way of staining nuclear DNA for densitometric quantification (Hardie et al., 2002).

Exfoliative cytology is a painless, non-invasive procedure, and is very well accepted by patients as it causes little discomfort (Nayar et al., 2003). Whilst oral exfoliative cytology is popular as an oral cancer-screening tool, its potential as a diagnostic mode is still challenged. There is an increasing need to refine exfoliative cytology techniques for it to be utilized as a diagnostic test. Hence, in this study we used a combination of Feulgen stain and the differentiating stain of Papanicaolou stain Orange G6 (OG-6) and Eosin Azure 50 (EA-50) for cytological evaluation of oral keratinocytes. The cytomorphometric features of keratinocytes obtained from the mucosa of tobacco abusers and from OSCC lesion were analyzed and compared. Thereby, an attempt was made to identify cytomorphometric features of keratinocytes that could signify an impending malignant transformation.

\section{Materials and Methods}

The material for the study was oral smears obtained from the mucosa of tobacco users, OSCC patients and from apparently healthy individuals. The individuals for the study and control groups were the patients who reported to the Department of Oral Medicine \& Radiology, Manipal College of Dental Sciences and Department of Radiotherapy \& Oncology, Kasturba Medical College,
Manipal.

\section{Patient selection}

Twenty patients who practice chewing tobacco or smoking or both, but who had no clinically visible alteration in the oral mucosa (criteria set forth by Einstein et al. (2005) was followed), served as the tobacco-using group. Twenty cases of clinically diagnosed oral carcinomas (that were histopathologically confirmed as squamous cell carcinomas) were also selected. Finally, twenty healthy individuals who were non-smokers and non-alcoholic, and with clinically normal oral mucosa were served as controls.

Exclusion criteria: Anemic patients, patients with history of any recent systemic disease and those on medication were excluded from the study to avoid cellular changes associated with these conditions.

Ethical clearance was obtained from the institutional ethical committee to undertake the study. A performa was framed and all the relevant information of each patient was recorded. A thorough head, neck and oral cavity examination was performed for all patients using universal precautions. After thorough examination, the procedure was described to the patient and written consent was obtained. Patients were asked to rinse their mouth to remove debris and the surface was cleaned with a swab dampened with saline. Oral smears were then collected with a Cytobrush ${ }^{\circledR}$ plus GT (Medscan Medical AB, Molmo, Sweden) (Figure 1).

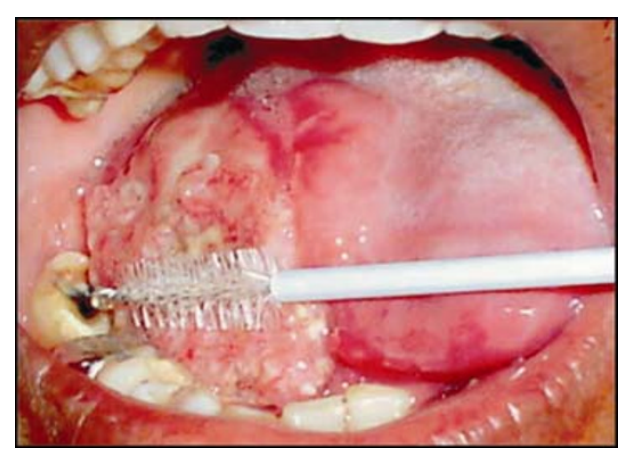

Figure 1 Collection of exfoliated cells from an OSCC lesion

For the clinically diagnosed cases of carcinoma, smears were obtained from on and around the 
lesion. In addition, smears were also collected from the apparently normal mucosa on the side contra-lateral to the lesion. For the controls, smears were taken only from the buccal mucosa. In order to gather cells from all the layers of the epithelium, a moderate pressure was applied while taking the smear. Cells that were collected on the Cytobrush ${ }^{\circledR}$ plus GT were spread evenly on a clean, frosted end, labeled glass slide. The smears were immediately fixed with 1:1 mixture of $95 \%$ ethanol + ether and carbowax for one hour. Two smears were prepared for each of the cases and controls. Once the smears were selected for the study, they were categorized into four groups (Table 1).

Table1 The categories of oral smears that were evaluated

\begin{tabular}{llc}
\hline Categories & \multicolumn{1}{c}{ Smears obtained from } & Sample size \\
\hline $\begin{array}{l}\text { Study group } \\
\text { Group I }\end{array}$ & $\begin{array}{l}\text { Mucosa of tobacco users } \\
\text { (chewing and smoking) }\end{array}$ & 20 \\
Group II & OSCC-lesion & 20 \\
Control group & & 20 \\
Group III & Apparently normal mucosa & \\
& of the contra-lateral side of & \\
& the OSCC lesions & \\
Group IV & Oral mucosa of healthy & 20 \\
& individuals \\
\hline
\end{tabular}

\section{Histochemical staining}

All the smears were stained with Feulgen reaction and counterstained with OG-6, EA-50. The slides were placed in $80 \%$ isopropyl alcohol for 2 changes of 15 minutes each to remove coating of carbowax. The sections were then brought to water through different grades of alcohol. The slides were rinsed in $1 \mathrm{~mol} \cdot \mathrm{L}^{-1} \mathrm{HCl}$ for 1 minute at room temperature. The slides were placed in $1 \mathrm{~mol} \cdot \mathrm{L}^{-1} \mathrm{HCl}$ for 10 minutes at $60^{\circ} \mathrm{C}$ and rinsed in $1 \mathrm{~mol} \cdot \mathrm{L}^{-1} \mathrm{HCl}$ for 1 minute at room temperature. Slides were transferred to Schiff's reagent and were allowed to remain for 45 minutes. The slides were rinsed in bisulphite solution thrice for 2 minutes each. Finally, the slides were rinsed well in distilled water for 10-12 minutes. To coun- terstain, slides were rinsed in $70 \%$ and $95 \%$ alcohol. Slides were placed in OG-6 for 1.5 minutes. Slides were rinsed in $95 \%$ alcohol twice. Slides were then placed in EA-50 for 1.5 to 2 minutes, rinsed in $95 \%$ alcohol twice and absolute alcohol twice, cleared in xylene, and mounted with D.P.X. The smears, when stained, had red-purple nuclei/ micronuclei or DNA component and cytoplasm that appeared in different colours ranging from orange to pink-red to blue-green depending on the degree of maturation (Figure 2).

\section{Cytomorphometric analysis}

All the stained smears were observed under a research light microscope.

Fifty cells with defined borders were randomly selected. Images of the cells under high power objective of $40 \times$ magnification and $10 \times$ magnification of eyepiece were captured with the help of a digital camera. The images were uploaded for computer analysis. The morphometric analysis was done using the Windows ${ }^{\circledR}$ based image analyzer software (ImageJ 1.34s) developed by National Institute of Health, USA and available at website http://rsb.info.nih.gov/ij/).

\section{Nuclear and cellular area}

For measurement, the nucleus and cell outline was traced on the screen and the software automatically calculated the cell area. The measurement was recorded in square microns (Figure 3).

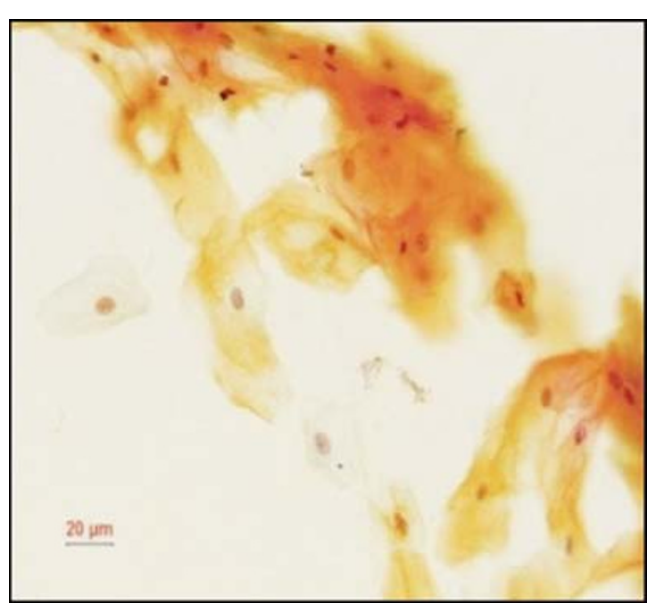

Figure 2 Cytological smear showing keratinocytes at different stages of maturation

Papanicolaou and Feulgen stain $(\times 10)$ 


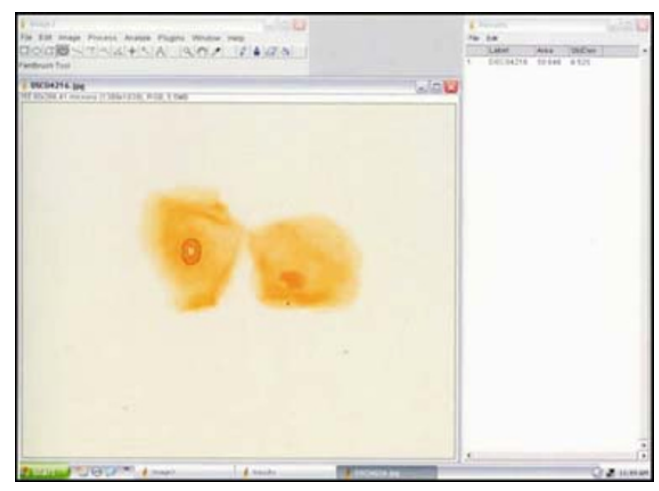

Figure 3 Measurement of cell area using the image analysis software program

\section{Nucleus and cell diameter}

Following calculations for the area the same cells were subjected to calculations of diameter. This was also measured in microns. The nuclear and cell diameter were calculated taking the mean of diameters in two perpendicular plane.

\section{Nuclear-to-cellular area ratio}

$$
\text { Nuclear }- \text { to }- \text { cellular area ratio }=\frac{\text { Nuclear area }}{\text { Cell area }}
$$

\section{Statistical analysis}

Since this study involves multiple groups, oneway ANOVA (Analysis of Variance) was used for comparing the parameters for multiple groups. Comparison of the mean nuclear and cellular area and diameter values between groups was made using multiple comparison test by Tukey-HSD procedure. The $P$-value $<0.05$ was considered to be significant.

\section{Results}

The mean values of nuclear area, nuclear diameter, cellular area, cellular diameter, nuclear-to-cellular area ratio of all four groups is shown in Figures $4-8$ respectively.

\section{Nuclear area in various study groups}

The mean nuclear area of keratinocytes in Group I (OSCC lesion) smears was much greater than that of the other three groups (Figure 4). The mean nuclear area of Group III and I was almost the same, while that of the normal oral mucosa was less (Figure 4). The Tukey HSD formula test showed a significant difference in the nuclear areas between all the groups except between Group I and Group III (Table 2).

\section{Nuclear Diameter in various study groups}

The mean value of the nuclear diameter of keratinocytes in group II smears were the highest when compared with those of the other three groups (Figure 5). The values of Group I and Group III smears were identical and the mean nuclear diameter value was the least for Group IV smears. Tukey HSD formula for pair wise comparison showed a significant difference in the mean values of between all the groups except between Group I and Group III (Table 2).

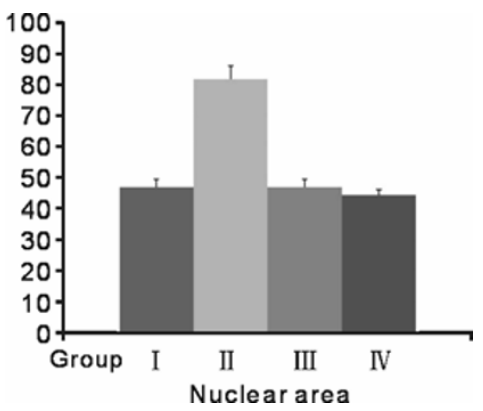

Figure 4 Mean value of the nuclear area among the four groups

Group I : $46.58 \pm 2.57$; Group II: $81.54 \pm 4.31$; Group III: $46.66 \pm 2.48$; Group IV: $44.10 \pm 1.71$.

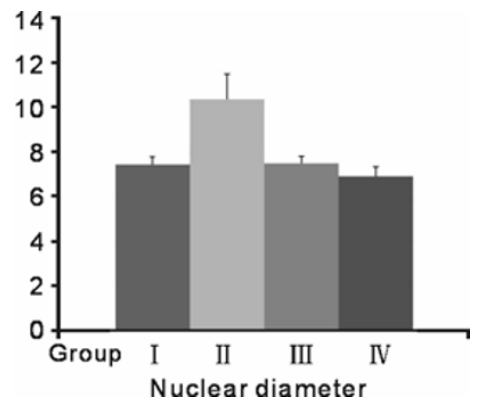

Figure 5 Mean value of the nuclear diameter among the four groups

Group I : $7.47 \pm 0.34$; Group II: $10.41 \pm 1.12$;

Group III: $7.51 \pm 0.33$; Group IV: $6.93 \pm 0.43$. 
Table 2 Comparison of the mean values of the nuclear area and the nuclear diameter among the four groups

\begin{tabular}{ccccccc}
\hline \multirow{2}{*}{ Groups compared } & \multicolumn{2}{c}{ Nuclear area } & & \multicolumn{2}{c}{ Nuclear diameter } \\
\cline { 3 - 4 } \cline { 6 - 7 } \cline { 5 - 6 } & & Mean difference & $P$ & & Mean difference & $P$ \\
\hline \multirow{2}{*}{ Group I } & Group II & 34.9683 & $0.0018^{*}$ & & 2.9410 & $0.001^{*}$ \\
& Group III & 0.0802 & 1.000 & & 0.0499 & 0.995 \\
& Group IV & 2.4757 & 0.044 & & 0.5378 & 0.049 \\
Group II & Group III & 34.8881 & $0.001^{*}$ & & 2.8911 & $0.001^{*}$ \\
& Group IV & 37.4440 & $0.001^{*}$ & & 3.4788 & $0.001^{*}$ \\
Group III & Group IV & 2.5559 & 0.035 & & 0.5877 & 0.026 \\
\hline
\end{tabular}

* Tukey HSD formula $P$-value-significant.

One way ANOVA-Nuclear area-F=751.016, $P=0.001$; Nuclear diameter- $\mathrm{F}=119.147, P=0.001$.

Table 3 Comparison of the mean values of the cellular area and cellular diameter among the four groups

\begin{tabular}{ccccccc}
\hline \multirow{2}{*}{ Groups compared } & \multicolumn{2}{c}{ Cellular area } & & \multicolumn{2}{c}{ Cellular diameter } \\
\cline { 3 - 4 } \cline { 6 - 7 } \cline { 6 - 7 } & & Mean difference & $P$ & & Mean difference & $P$ \\
\hline Group I & Group II & -336.8385 & $0.001^{*}$ & & -6.7748 & $0.001^{*}$ \\
& Group III & -24.3726 & 0.679 & & -0.6230 & 0.798 \\
& Group IV & -256.8137 & $0.001^{*}$ & & -4.8061 & $0.001^{*}$ \\
Group II & Group III & -312.4659 & $0.001^{*}$ & & -6.1518 & $0.001^{*}$ \\
& Group IV & -593.6522 & $0.001^{*}$ & & -11.5809 & $0.001^{*}$ \\
Group III & Group IV & -281.1863 & $0.001^{*}$ & & -5.4291 & $0.001^{*}$ \\
\hline
\end{tabular}

* Tukey HSD formula $P$-value-significant.

One way ANOVA-Cellular area-F=248.854, $P<0.001$, cellular diameter-F=96.7, $P<0.001$.

\section{Cellular area in various study groups}

The mean value of cellular area was greatest among the keratinocytes of Group IV smears. Group II smears contained keratinocytes with the lowest cellular area among all four groups (Figure 6). Group I smears showed keratinocytes with slightly larger mean cellular area values than those in Group III. Tukey HSD formula test for pair wise comparison showed a significant difference between all the groups except between Group I and Group III (Table 3).

\section{Cell diameter in various study groups}

The highest values of mean cell diameter of the keratinocytes was seen in Group IV smears (Figure 7). Group II smears had the lowest mean value for cell diameter. Group I and Group III smears had keratinocytes with similar mean cell diameter values. Tukey HSD formula for pair wise comparison revealed that results were very significant for comparison between all the groups except between Group I and Group III (Table 3).

\section{Nuclear-to-cellular area ratio in various study groups}

The mean value of the ratio between nuclear/ cellular area for Group II was the highest among the four groups (Figure 8). Group IV smears contained keratinocytes with the lowest mean nuclearto-cellular area ratio. There was a non-significant difference in the mean ratio between Group I and Group III. Tukey HSD formula showed significant result between all the groups except those between Group I and Group III (Table 4). 


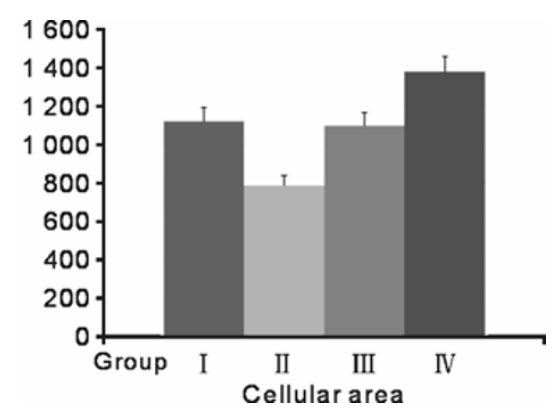

Figure 6 Mean value of the cellular area among the four groups

Group I : $1120 \pm 70.44$; Group II: $783.62 \pm 53.33$;

Group III: $1096 \pm 70.55$; Group IV: $1377.30 \pm 76.68$.

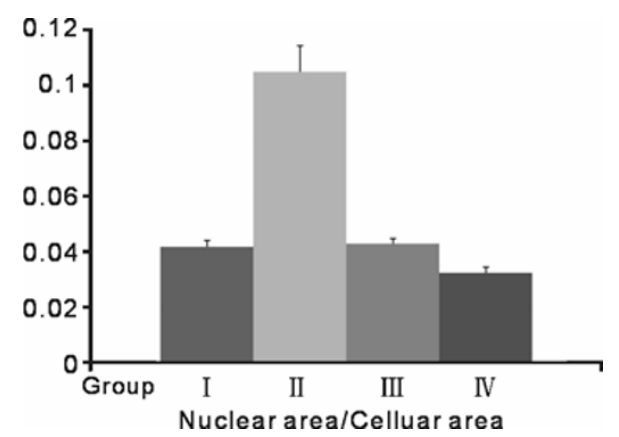

Figure 8 Mean value of the nuclear-cellular area ratio among the four groups

Group I : $0.0416 \pm 0.002$ 3; Group II: $0.1045 \pm 0.009$ 5; Group III: $0.0426 \pm 0.008$ 1; Group IV: $0.0322 \pm 0.0021$.

Table 4 Comparison of the mean values of the nuclear-cellular area ratio among the four groups

\begin{tabular}{cccc}
\hline Groups Compared & \multicolumn{2}{c}{ Mean difference in } \\
& & $\begin{array}{l}\text { nuclear/cell area } \\
\text { ratio }\end{array}$ & $P$ \\
\hline \multirow{2}{*}{ Group I } & Group II & 0.0629 & $0.001^{*}$ \\
& Group III & 0.0010 & 0.924 \\
& Group IV & 0.0094 & $0.001^{*}$ \\
Group II & Group III & 0.0620 & $0.001^{*}$ \\
Group III & Group IV & 0.0724 & $0.001^{*}$ \\
\hline
\end{tabular}

* Tukey HSD formula $P$-value-significant.

One way ANOVA-F $=521.2, P<0.001$.

\section{Discussion}

In the present study, we found that the mean nuclear area value of keratinocytes was higher in OSCC lesions when compared with those from the

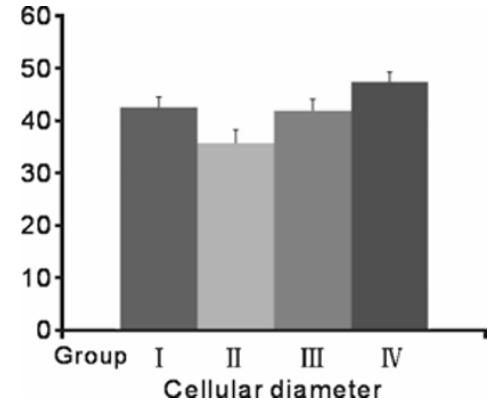

Figure 7 Mean value of cellular diameter among the four groups

Group I : $42.59 \pm 1.99$; Group II: $35.81 \pm 2.48$;

Group III: $41.96 \pm 2.21$; Group IV: $47.39 \pm 1.90$.

mucosa of tobacco users. These values in keratinocytes in the control normal mucosa, however, were lower than those of the tobacco abusers. Malignant cells display a significant increase in mean nuclear area (Cowpe, 1984). The increase in the nuclear size is related to an increase in the nuclear contents required for replication (Frost, 1997).

Conversely, we found that the mean value for the cellular area and cellular diameter of keratinocytes obtained from the mucosa of tobacco abuser was greater than those of OSCC lesions. But these values were higher in keratinocytes of the normal mucosa. Frost et al. stated that in cells with increased activity the ability of cytoplasm to mature diminishes (Frost, 1997). Additionally, the amount of cytoplasm the cell makes in relation to the nucleoplasm is less. Decrease in the cellular diameter and increase in the nuclear size are two significant changes that occur in actively proliferating cell (Cançado et al., 2004). Accordingly, we observed that with the progression from normal to malignancy, the nuclear dimensions increased and cellular dimensions decreased.

Next, we found that the cytomorphometric characteristics of keratinocytes of the clinically normal mucosa adjacent to the OSCC lesions and those of the tobacco abusers were not significantly different. The mean value for cell diameter and cellular area of these keratinocytes was greater than that of the malignant epithelial cells. However, the mean value for nuclear area and diameter was less than those of the malignant lesion tissue. But keratinocytes in both these groups had cytomorphological features that were significantly different from those of the normal 
mucosa in healthy individuals. Ogden et al. while assessing evidence of field change by quantitative cytomorphometric analysis of smears from normal buccal mucosa in oral cancer patients and cancer free patients using image analysis system found a statistically significant reduction in cytoplasmic area but no change in nuclear area (Ogden et al., 1990). They believed that this could be the first evidence for true field change in oral cancer at light microscopic level.

Freitas et al. used image analysis techniques to compare the nucleus area, cytoplasm area and nuclear-cellular area ratio between oral mucosa cells from 10 patients with oral carcinoma and 10 healthy subjects (Freitas et al., 2003). They concluded that neither cytoplasm area and nucleus area nor nuclear-cellular area ratio differ between the oral mucosa of oral carcinoma patients and healthy patients. In this study, however, the nuclear-cellular area ratio of keratinocytes of the oral carcinoma lesions was significantly greater than those of the mucosa of normal, healthy individuals.

The cytobrush is a convenient instrument capable of sampling less accessible oral sites. In addition, the cytobrush pulls together an adequate number of cells and allows uniform dispersion of the cells on a micro-slide which facilitates an accurate cytopathologic diagnosis (Ogden et al., 1992; Jones et al., 1994). Hence, to capitalize on these valuable benefits, we used a Cytobrush ${ }^{\circledR}$ plus GT to acquire the oral smears for the study.

The differentiating stain of PAP, OG-6 and EA-50 provides a subtle range of green, blue and pink hues to the cell cytoplasm according to the amount of keratin content and the maturation pattern of the cell (Atkins et al., 1956). Feulgen reaction is both specific and stoichiometric for DNA and imparts a pinkish red color to the nucleus. The intensity of staining can be correlated with the nuclear DNA distribution (Hardie et al., 2002). Quantitative analysis of nuclear DNA-content based on Feulgen reaction was found to reflect on the total chromosomal content (Bibbo et al., 1997). DNA ploidy analysis of Feulgen stained smears has shown $90.9 \%$ of diploid and $9.1 \%$ aneuploid DNA patterns in normal mucosa, whereas in malignant lesions the aneuploidy rate was $16.7 \%$ and the diploid pattern was indicated for $83.3 \%$
(Pektas et al., 2006).

Feulgen stained malignant cells display an elevation in nuclear area corresponding to the abnormality in the DNA profiles that is not always evident in Papanicoloau stained smears (Cowpe et al., 1985; Cowpe et al., 1995). The biological activity of any cell is best reflected in the nucleus and the functional activity in the cytoplasm. Hence, in this study a combination of Feulgen stain (nuclear stain) and OG-6 and EA-50 (cytoplasmic differentiation stain) was used to derive optimal information on the cellular events taking place in the keratinocytes.

In summary, this study supports and extends the view that cytomorphometric evaluation of keratinocytes can serve as a useful diagnostic adjunct for early detection of oral cancer. An increase in the nuclear size and decrease in the cellular size are characteristics of malignant keratinocytes. Evaluation of a greater number of cases is essential to establish the cut-off values of these parameters (i.e., sensitivity and specificity) that can be used as definitive indicators.

\section{Conclusion}

The basic defect of any cell alteration begins at the molecular level, and triggers a series of reactions which affect the entire cell system. This is ultimately reflected in the cellular morphology. Cytomorphometric analysis of smears can be carried out regularly to detect alterations in the cellular and nuclear dimensions especially among tobacco users. Cytomorphometric assessment of the keratinocytes will assist in monitoring the status of the mucosa. Further, as the acceptance in the reliability of measurable values increases, this method can also aid in motivating individuals to withdraw from tobacco use.

\section{Reference}

Atkins NB, Richards BM (1956). Deoxyribonuclei acid in human tumours as measured by microspectrometry of Feulgen stain: a comparison of tumours arising at different sites. Br J Cancer, 10(4): 769-787.

Bancroft JD, Stevens A. Theory and practice of histological techniques. 4rd ed. New York: Churchill 
Livingstone, 1996.

Bibbo M. Comprehensive cytopathology. 2nd ed. Pennsylvania: WB Saunders Company, 1997.

Boyle P, Macfarlans GJ, Maisonneuve P, Zheng T, Scully C, Tedsceo B (1990). Epidemiology of mouth cancer in 1989: a review. JR Soc Med, 83(11): 724-730.

Cançado RP, Yurgel LS, Filho MS (2004). Comparative analysis between the smoking habit frequency and the nucleolar organizer region associated protein in exfoliative cytology of smokers' normal buccal mucosa. Tob Induc Dis, 15, 2(1): 43-49.

Cowpe JG (1984). Quantitative exfoliative cytology of normal and abnormal oral mucosal squames: preliminary communication. $J R$ Soc Med, 77(11): 928931.

Cowpe JG, Longmore RB (1981). Nuclear area and Feulgen DNA content of normal buccal mucosal smear. J Oral Pathol, 10(2): 81-86.

Cowpe JG, Longmore RG, Green MW (1985). Quantitative exfoliative cytology of normal oral squames: an age, site and sex-related survey. $J R$ Soc Med, 78(12): 9951004.

Cowpe JG, Ogden GR, Thompson M (1995). Comparison of nuclear area measurements carried out on normal and dysplastic oral smears. Anal Quant Cytol Histol, 17(4): 241-246.

Einstein TB, Sivapathasundharam B (2005). Cytomorphometric analysis of the buccal mucosa of tobacco users Ind J Dent Res, 16(2): 42-46.

Freitas MD, Garcia-Garcia A, Carneiro JLM (2003). Exfoliative cytology of the oral mucosa: a cytomorphometric comparison of healthy oral mucosa in oral cancer patients and healthy subjects. Revista Brasileira Pathologia Oral, 2(4): 2-6.

Frost JK (1997). Pathologic processes affecting cells from inflammation to cancer. //Bibbo M, ed. Comprehensive cytopathology. 2 ed. Philadelphia: WB Saunders company, p 845.

Hardie DC, Gregory TR, Hebert PD (2002). From pixels to picograms: a beginners' guide to genome quantification by Feulgen image analysis densitometry. $J$
Histochem Cytochem, 50(6): 735-749.

Jones AC, Pink FE, Sandow PL, Stewart CM, Migliorati CA, Baughman RA (1994). The Cytobrush Plus cell collector in oral cytology. Oral Surg Oral Med Oral Pathol, 77(1): 95-99.

Miller SC, Soberman A, Stahl SS (1951). Study of cornification of oral mucosa in young male adults. $J$ Dent Res, 30(1): 4-11.

Montgomery PW, von Hamm E (1951). Study of exfoliative cytology in patients with carcinoma of the oral mucosa. $J$ Dent Res, 30(3): 308-313.

National Cancer Institute. SEER Cancer Statistics Review: 1973-1998. [WWW document]. URL http://seer.cancer. gov/csr/1973_1998/index.html.

Nayar AK, Sundharam BS (2003). Cytomorphometric analysis of exfoliated normal buccal mucosa cells. Indian J Dent Res, 14(2): 87-93.

Ogden GR, Cowpe JG (1990). Quantitative exfoliative cytology of normal buccal mucosa: effect of smoking. J Oral Pathol Med, 19(2): 53-55.

Ogden GR, Cowpe JG, Green MW (1992). Cytobrush and wooden spatula for oral exfoliative cytology - A comparison. Acta cytol, 36(5): 706-710.

Parkin DM, Bray F, Ferlay J, Pisani P (2001). Estimating the world cancer burden: 2000 Globocan. Int J Cancer, 94(2): 153-156.

Pektas ZO, Keskin A, Günhan O, Karslioğlu Y (2006). Evaluation of nuclear morphometry and DNA ploidy status for detection of malignant and premalignant oral lesions: quantitative cytologic assessment and review of methods for cytomorphometric measurement. Int $J$ Oral Maxillofac Surg, 64(4): 628-635.

Ramesh T, Mendis BR, Ratnatunga N, Thattil RO (1999). The effect of tobacco smoking and of betel chewing with tobacco on the buccal mucosa: a cytomorphometric analysis. J Oral Pathol Med, 28(9): 385-388.

Sivapathasundharam B, Kalasagar MY (2004). Yet another article on exfoliative cytology. J Oral Maxillofac Pathol, 8(2): $54-57$.

Sugerman PB, Savage NW (1996). Exfoliative cytology in clinical oral pathology. Aust Dent J, 41(2): 71-74.

*Corresponding author: Monica Charlotte Solomon

Address: Department of Oral Pathology, Manipal College of Dental Sciences, Manipal University, Manipal, India Tel: $918202922204 \quad$ Fax: 918202571966 E-mail: monicasolomon@yahoo.com 